\title{
INSPECTION PROGRAM DEVELOPMENT FOR AN AIRCRAFT FLEET AND AN AIRLINE ON THE BASIS OF THE ACCEPTANCE FATIGUE TEST RESULT
}

\author{
Yuri Paramonov ${ }^{1}$, Sergey Tretyakov ${ }^{2}$, Maris Hauka ${ }^{3}$ \\ Riga Technical University, Aeronautical Institute, \\ Lomonosova st. 1A/1, Riga, LV-1019 Latvia \\ IPhone: +37127866270.E-mail: yuri.paramonov@gmail.com \\ ${ }^{2}$ Phone: +371 26732434.E-mail: sergejs.tretjakovs@gmail.com \\ ${ }^{3}$ Phone: +371 26276057. E-mail:maris.hauka@gmail.com
}

\begin{abstract}
An inspection interval planning is considered in order to limit the probability of any fatigue failure (FFP) in a fleet of $N$ aircraft (AC) and to provide an economical effectiveness of airline (AL) under the limitation of fatigue failure rate (FFR). A solution of these two problems is based on the processing of the result of acceptance fatigue test of a new type of aircraft. During this test an estimate of the parameter $\hat{\theta}$, of a fatigue crack growth trajectory has been obtained. If the result of this acceptance test is too bad then this new type of aircraft will not be used in service. A redesign of this project should be done. If the result the acceptance test is pretty good then the reliability of the aircraft fleet and the airline will be provided without inspections. For this strategy there is a maximum of FFP (a maximum of FFR) as a function of an unknown parameter $\theta$. This maximum can be limited by the use of the offered here procedure of the choice of the inspection number. The economic effectiveness of the AL operation is considered using the theory of Markov process with rewords.
\end{abstract}

Keywords: Monte Carlo, Markov chains, Minimax, inspection program and approval test, fleet reliability, economic effectiveness

\section{Introduction}

We assume that in the interval $\left(T_{d}, T_{c}\right)$, where $T_{d}$ is a random time when the fatigue crack becomes detectable (the corresponding crack size $a\left(T_{d}\right)=a_{d}$ ) and $T_{c}$ is a random time when the crack reaches its critical size (the corresponding crack size $a\left(T_{c}\right)=a_{c}$ ), the size of the crack can be approximated by the equation $a(t)=\alpha \exp (Q t)$. Then we have:

$T_{c}=\left(\log a_{c}-\log \alpha\right) / Q=C_{c} / Q, T_{d}=\left(\log a_{d}-\log \alpha\right) / Q=C_{d} / Q$.

For the calculation of the probability of the fatigue crack detection during an inspection we need to know the distribution of the vector $\left(T_{d}, T_{c}\right)$. For the considered numerical example here (see the 5 -th section) we study the simplest case: a random variable (rv), $\log (Q)$, has a normal distribution with an unknown mean $\theta$ and the known standard deviation. And we assume that if $\theta$ is known then the distribution of the vector $\left(T_{d}, T_{c}\right)$ is also known. The estimate $\hat{\theta}$ of the parameter $\theta$ can be obtained by the regress analysis of the result of the fatigue test of $\mathrm{AC}$ of the same type in the laboratory (i.e. processing the observations of the fatigue crack: pairs $\left.\{\text { (time, fatigue crack size })_{i}, \mathrm{i}=1, \ldots, \mathrm{m}\right\}$, where $m$ is the number of the fatigue crack observations.

\section{Calculation of the probability of a fatigue failure of one aircraft, the fatigue failure rate and the economical effectiveness of the airline for the known $\theta$}

For the known $\theta$, there are two decisions: 1) the aircraft is good enough and the operation of this aircraft type can be allowed, 2) the operation of the new type of AC is not allowed and a redesign of AC should be done. In the case of the first decision, the vector $t=\left(t_{1}, \ldots, t_{n}\right)$, where $t_{i}$ is the time moment of 
$i$-th inspection, should also be defined. If $\theta$ is known the different rules can be offered for the choice of the structure of the vector $t: 1)$ every interval between the inspections is equal to the constant $\Delta_{t}=t_{S L} /(n+1)$, where $t_{S L}$ is the aircraft specified life (SL) (the retirement time), $n$ is the number of inspections, 2) the conditional probabilities of a failure in every interval is equal to the same value $P\left(T_{C}<t_{S L}\right) /(n+1) \ldots$ In this paper we assume the first type of the choice and the vector $t$ is defined by the fixed $t_{S L}$ and the choice of $n$.

For the substantiation of the choice of the inspection number we should know FFP of AC and FFR and the gain of AL as the functions of $n$. For this purpose the process of an operation of AC can be considered as an absorbing Markov chain $(\mathrm{MCh})$ with $(n+4)$ states. The states $E_{1}, E_{2}, \ldots, E_{n+1}$ correspond to the AC operation in the time intervals $\left[t_{0}, t_{1}\right),\left[t_{1}, t_{2}\right), \ldots,\left[t_{n}, t_{S L}\right)$. States $E_{n+2}, E_{n+3}$ and $E_{n+4}$ are the absorbing states: AC is discarded from the service when the SL is reached or fatigue failure (FF), or fatigue crack detection (CD) take place.

\begin{tabular}{|c|c|c|c|c|c|c|c|c|c|c|}
\hline $\mathrm{E}_{1}$ & 0 & $\mathrm{u}_{1}$ & 0 & $\ldots$ & 0 & 0 & 0 & 0 & $\mathrm{q}_{1}$ & $\mathrm{v}_{1}$ \\
\hline $\mathrm{E}_{2}$ & 0 & 0 & $\mathrm{u}_{2}$ & $\ldots$ & 0 & 0 & 0 & 0 & $\mathrm{q}_{2}$ & $\mathrm{v}_{2}$ \\
\hline $\mathrm{E}_{3}$ & 0 & 0 & 0 & $\ldots$ & 0 & 0 & 0 & 0 & $\mathrm{q}_{3}$ & $\mathrm{v}_{3}$ \\
\hline$\ldots$ & $\ldots$ & $\ldots$ & $\ldots$ & $\ldots$ & $\ldots$ & $\ldots$ & $\ldots$ & $\ldots$ & $\ldots$ & $\ldots$ \\
\hline $\mathrm{E}_{\mathrm{n}-1}$ & 0 & 0 & 0 & $\ldots$ & 0 & $\mathrm{u}_{\mathrm{n}-1}$ & 0 & 0 & $\mathrm{q}_{\mathrm{n}-1}$ & $\mathrm{v}_{\mathrm{n}-1}$ \\
\hline $\mathrm{E}_{\mathrm{n}}$ & 0 & 0 & 0 & $\ldots$ & 0 & 0 & $\mathrm{u}_{\mathrm{n}}$ & 0 & $\mathrm{q}_{\mathrm{n}}$ & $\mathrm{v}_{\mathrm{n}}$ \\
\hline $\mathrm{E}_{\mathrm{n}+1}$ & 0 & 0 & 0 & $\ldots$ & 0 & 0 & 0 & $\mathrm{u}_{\mathrm{n}+1}$ & $\mathrm{q}_{\mathrm{n}+1}$ & $\mathrm{v}_{\mathrm{n}+1}$ \\
\hline $\begin{array}{c}\mathrm{E}_{\mathrm{n}+2} \\
(\mathrm{SL})\end{array}$ & 0 & 0 & 0 & $\ldots$ & 0 & 0 & 0 & 1 & 0 & 0 \\
\hline $\begin{array}{c}\mathrm{E}_{\mathrm{n}+3} \\
(\mathrm{FF})\end{array}$ & 0 & 0 & 0 & $\ldots$ & 0 & 0 & 0 & 0 & 1 & 0 \\
\hline $\begin{array}{c}\mathrm{E}_{\mathrm{n}+4} \\
(\mathrm{CD})\end{array}$ & 0 & 0 & 0 & $\ldots$ & 0 & 0 & 0 & 0 & 0 & 1 \\
\hline
\end{tabular}

Figure 1. Probability matrix $\mathrm{P}_{\mathrm{AC}}$

In the corresponding transition probability matrix, $P_{A C}$, let $v_{i}$ be the probability of a crack detection during the inspection number $i$, let $q_{i}$ be the probability of the failure in the service time interval $t \in\left(t_{i-1}, t_{i}\right)$, and let $u_{i}=1-v_{i}-q_{i}$ be the probability of the successful transition to the next state. In our model we also assume that an aircraft is discarded from the service at $t_{S L}$ even if there are no any cracks discovered by the inspection at the time moment $t_{S L}$. This inspection at the end of $(n+1)$ -th interval (in state $E_{n+1}$ ) does not change the reliability but it is made in order to know the state of the aircraft (whether there is a fatigue crack or there is no fatigue crack). It can be shown that $u_{i}=P\left(T_{d}>t_{i} \mid T_{d}>t_{i-1}\right), q_{i}=P\left(t_{i-1}<T_{d}<T_{c}<t_{i} \mid T_{d}>t_{i-1}\right), \quad v_{i}=1-u_{i}-q_{i}, i=1, \ldots, n+1$. In the three last lines of the matrix $P_{A C}$ there are three units in the matrix diagonal because the states $E_{n+2}$, $E_{n+3}$ and $E_{n+4}$ are the absorbing states. All the other entries of this matrix are equal to zero. 


\begin{tabular}{|c|c|}
\hline $\mathrm{Q}$ & $\mathrm{R}$ \\
\hline 0 & $\mathrm{I}$ \\
\hline
\end{tabular}

The structure of the considered matrix can be described in the following way: $P_{A C}=[Q R ; 0 I]$, where in the second line of this structure the matrix 0 is the sub matrix of zeros, $I$ is the sub matrix of identity corresponding to the absorbing states of the matrix $P$. Then the matrix of the probabilities of absorbing in the different absorbing states for the different initial transient states $B=(I-Q)^{-1} R$. The failure probability of a new $\mathrm{AC}$ is equal to $p_{f}=a B b$, where the vector row $a=(1,0, \ldots, 0)$ means that all the aircraft begin an operation within the first interval (state $\left.E_{1}\right)$, the vector column $b=(0,1,0)^{\prime}$. We need also to know the mean number of steps of MCh up to the absorption $E\left(T_{A C}\right)=a(I-Q)^{-1} c$, where $c=(1, \ldots, 1)^{\prime}$ is the vector-column. The mean life of the aircraft will be equal to $\Delta_{t} E\left(T_{A C}\right)$.

In the corresponding matrix for the operation process of AL, $P_{A L}$, the states $E_{n+2}, E_{n+3}$ and $E_{n+4}$ are not absorbing but correspond to the return of $\mathrm{MC}$ to the state $E_{1}$ (there are the units in the first column of the last 3 rows of the matrix $P_{A L}$; the AL operation returns to the first interval). The other lines of $P_{A C}$ and $P_{A L}$ are the same.

Using the definition of $P_{A L}$ we can get the vector of stationary probabilities, which is defined by the equation system: $\pi P_{A L}=\pi, \sum_{i=1}^{n+4} \pi_{i}=1$ and the airline gain $g(n)=\sum_{i=1}^{n+4} \pi_{i} g_{i}(n)$ where:

$g_{i}(n)=\left\{\begin{array}{c}a_{i} u_{i}+b_{i} q_{i}+c_{i} v_{i}, i=1, \ldots, n+1 \\ d_{i}, i=n+2, \ldots, n+4\end{array}\right.$.

$a_{i}$ is the reward defined by the successful transition from one operation interval to the following one and the cost of one inspection; $b_{i}, c_{i}$ and $d_{i}$ correspond to the transition to the states $E_{n+3}(\mathrm{FF}), E_{n+4}(\mathrm{CD})$ and then to the state $E_{1}$ (the "cost" of FF of $\mathrm{AC}$, fatigue crack detection, acquisition of the new AC). Let us note that $L_{j}=1 / \pi_{j}$ defines the mean step number to return to the same state $E_{j}$. So $\lambda_{f}=\pi_{n+3} / \Delta_{t}$ is the FFR. Let us note that in the considered case the same value can be calculated in another way. This value is equal to the ratio of the aircraft failure probability, $p_{f}$, to the mean life of the new aircraft: $\lambda_{f}=p_{f} / \Delta_{t} E\left(T_{A C}\right)$ (recall, that $\Delta_{t} E\left(T_{A C}\right)$ is the mean time of the renewal operation of AL in the first interval). If $\theta$ is known we calculate the gain as the function of $n, g(n, \theta)$, and choose the number $n_{g}$ corresponding to the maximum of gain: $n_{g}(\theta)=\arg \max _{n} g(n, \theta)$. Then we calculate FFR as the function of $n, \lambda_{f}(n, \theta)$, and choose $n_{\lambda}$ in such a way that for any $n \geq n_{\lambda}$ the function $\lambda_{f}(n, \theta)$ will be equal or less than some value $\lambda: n_{\lambda}(\lambda, \theta)=\min \left\{n: \lambda_{f}(n, \theta) \leq \lambda\right.$, for all $\left.n \geq n_{\lambda}(\lambda, \theta)\right\}$. And finally we choose $n=n_{g \lambda}(\lambda, \theta)=\max \left(n_{g}, n_{\lambda}\right)$.

\section{Probability of any fatigue failure in the fleet of aircraft for the known $\theta$}

We consider the case when the operation of all $\mathrm{N}$ aircraft will be stopped if any fatigue crack will be detected. In order to limit the probability of a fatigue failure in the fleet (FFPN), it is enough to find at least one fatigue crack before a failure of any aircraft in the fleet takes place. The corresponding 
probability is equal to the expected value of the random variable $P_{f N W}=(1-w)^{R}$, where $w$ is a human factor: a probability, that a planned inspection will be done, $R$ is the total random number of inspections before the first failure in the whole fleet. Let $t_{k}^{+}, t_{k-1}^{+}<t_{k}^{+}, t_{0}^{+}=0$ be the "calendar" time moment when $k$-th aircraft begin the service, $T_{d k}^{+}=t_{k}^{+}+T_{d k}, T_{c k}^{+}=t_{k}^{+}+T_{c k}, \mathrm{k}=1,2, \ldots, N$ be the random calendar time moments when fatigue crack can be discovered and fatigue failure of AC takes place correspondingly, see Fig. 2. And let $K_{S L}=\left\{k: T_{c k}<t_{S L}, k=1,2, \ldots, N\right\}$ be a set of indexes of the aircraft, the failure of which can take place, if an inspection does not take place, $T_{f}^{+}=\min \left\{T_{f k}^{+}: k \in K_{S L}\right\}, \quad T_{f k}^{+}=\min \left\{T_{c k}^{+}, T_{f}^{+}\right\}, k \in K_{S L}, \quad R=\sum_{k \in K_{S L}} R_{k}, \quad$ where $R_{k}=\max \left(\left\{\left[\left(T_{f k}^{+}-t_{k}^{+}\right) / \Delta_{t}\right]-\left[\left(T_{d k}^{+}-t_{k}^{+}\right) / \Delta_{t}\right]\right\}, 0\right), k \in K_{S L}$, is the random inspection number of $k$-th aircraft from the set $K_{S L}$ if inspection interval $\Delta_{t}=t_{S L} /(n+1)$ (it is supposed a specific "calendar" schedule of the inspections for each aircraft: , $i=1,2, \ldots, n+1, k \in K_{S L}$ )

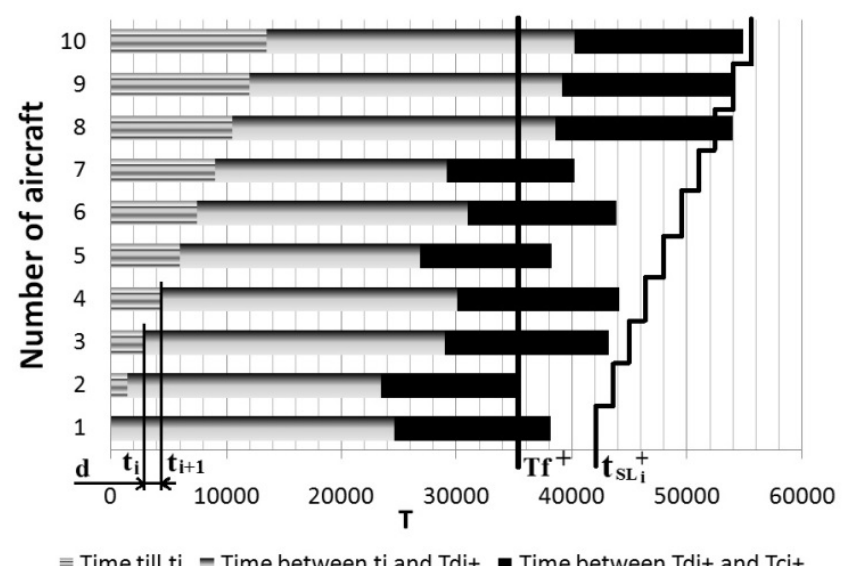

Figure 2. Inspection of $\mathrm{N}$ aircraft

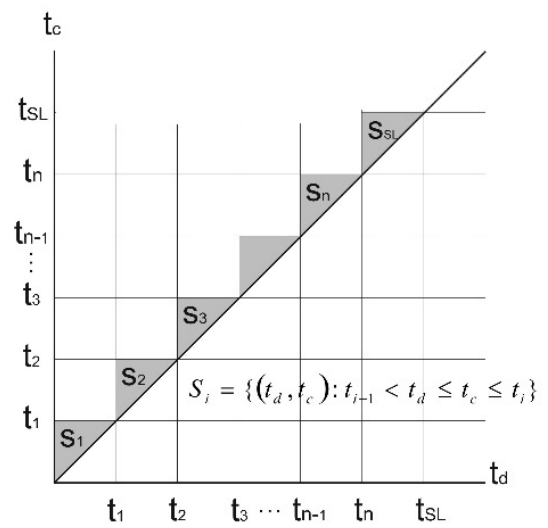

Figure 3. Example of set of sets $S_{Z, i}$

Random variable $Q$ is the speed of the fatigue crack growth in the logarithm scale. It has a specific realization for each aircraft and $Q_{1}, \ldots, Q_{N}$ are independent random variables. So, a mean value of a random probability of failure in the fleet

$$
E\left(P_{f N W}\right)=p_{f N W}(n, \theta)=\int_{-\infty}^{\infty} \ldots \int_{-\infty}^{\infty}(1-w)^{r(q)} d F_{Q_{i}}\left(q_{1}\right) \ldots d F_{Q_{N}}\left(q_{N}\right),
$$


where $q=\left(q_{1}, \ldots, q_{N}\right), r(q)$, is realization of $\mathrm{rv} R$. For a large number $N$ the Monte Carlo method is appropriate for the calculation of $p_{f N W}$. If this function is known then the number of the inspections, $n(p, \theta)$, required to limit the FFPN by value $p$ is defined by the function $n(p, \theta)=\min \left(r: p_{f N W}(r, \theta) \leq p\right.$ for all $\left.r>n(p, \theta), r=1,2, \ldots\right)$

\section{Solution for unknown $\theta$}

First, we consider the problem of the limitation of FFP1 in an operation of one AC with the human factor $w=1$. This means that if there is a detectable fatigue crack, then during the inspection we see it with probability 1 and the limitation of FFP1 of AC is provided by the choice of a specific p-set function, Paramonov et al (2011). Let us take into account that the operation of a new type of aircraft will not take place if the result of the acceptance fatigue test in the laboratory is "too bad" (previously, the redesign of a new type of AC should be done). We say that in this case the event $\hat{\theta} \notin \Theta_{0}, \Theta_{0} \subset \Theta$, takes place (for example, $\hat{\theta} \notin \Theta_{0}$ if fatigue life $T_{C}$ is lower than some limit; or $n(p, \hat{\theta})$ is too large,...). Let us define some set function

$S\left(\Theta_{0}, n\right)=\left\{\begin{array}{l}\bigcup_{i=1}^{n+1} S_{i}(n) \text { if } \hat{\theta} \in \Theta_{0}, \\ \varnothing, \text { if } \hat{\theta} \notin \Theta_{0}\end{array}\right.$

where $S_{i}=\left\{\left(t_{d}, t_{c}\right): t_{i-1}<t_{d}, t_{c} \leq t_{i}\right\}, t_{i}=i t_{S L} /(n+1), i=1, \ldots, n+1 ; \varnothing$ is an empty set.

It can be shown that for a very wide range of the definition of the set $\Theta_{0}$ and the requirements to limit FFP1 by the value $p^{*}$, where $\left(1-p^{*}\right)$ is the required reliability, there is a preliminary "designed" choice of the allowed FFP1, $p_{f D}$, such that the corresponding set function $S\left(\Theta_{0}, n\left(p_{f D}, \hat{\theta}\right)\right)$ is $p$-set function of the level $p^{*}$ for the vector $Z=\left(T_{d}, T_{c}\right): \sup _{\theta} \sum_{i=1}^{n+1} P\left(Z \in S_{i}\left(n\left(p_{f D}, \theta\right)\right)=p^{*}\right.$. This means that FFP1 will be limited by the value $p^{*}$ for any unknown $\theta \in \Theta$.

In a similar way, there is such $\lambda_{f D}$ that the choice of $n=n_{g \lambda}\left(\lambda_{f D}, \hat{\theta}\right)$ provides the required limitation of the expected value of FFR, $E\left(\lambda_{f}\left(\hat{\theta}, \lambda_{f D}, \Theta_{0}\right)\right)$, by a some allowed value FFR, $\lambda^{*}$, for every unknown $\theta \in \Theta$. For the requirement of a high reliability, the choice of the inspection number will be defined by the limitation of FFR but for a very high "cost" of FF of AC the choice will be defined by the maximum of the gain.

Now, we consider the reliability of the fleet of $\mathrm{N} \mathrm{AC}$ when there is information exchange and the operation of all aircraft is stopped if any fatigue crack is found during the inspection of any AC and, as it has been mentioned, in order to prevent a failure in the fleet, it is enough to find at least one fatigue crack before a failure of any aircraft in the fleet takes place. Let us define a multiple set function:

$$
S^{+}\left(\Theta_{0}, n\right)=\bigcup_{k \in K_{S L}} S_{k}^{+}\left(\Theta_{0}, n\right)
$$

where

$$
\begin{aligned}
& S_{k}^{+}\left(\Theta_{0}, n\right)=\left\{\begin{array}{l}
\bigcup_{i=1}^{n+1} S_{i, k}^{+}(n) \text { if } \hat{\theta} \in \Theta_{0}, \\
\varnothing, \text { if } \hat{\theta} \notin \Theta_{0},
\end{array}\right. \\
& S_{i k}^{+}=\left\{\left(t_{d k}^{+}, t_{c k}^{+}\right): t_{(i-1) k}<t_{d k}, t_{c k} \leq t_{i k}\right\}, \quad t_{i k}^{+}=t_{k}^{+}+t_{i}, \quad t_{i}=i t_{S L} /(n+1), \quad i=1, \ldots, n+1, k=1,2, \ldots, N .
\end{aligned}
$$

Again, it can be shown that for a very wide range of the definition the set $\Theta_{0}$ and the requirements to the 
limit FFPN by the value $p^{*}$, there is a preliminary "designed" choice of allowed FFPN, $p_{f D}$, such that the corresponding multiple set function $S^{+}\left(\Theta_{0}, n\left(p_{f D}, \hat{\theta}\right)\right)$ is $p$-set function of level $p^{*}$ for the set of vectors $\left\{Z_{k}^{+}, k \in K_{S L}\right\}$, where $Z_{k}^{+}=\left(T_{d k}^{+}, T_{f k}^{+}\right)$:

$v\left(p_{f D}\right)=p^{*}$, where

$v(p)=\sup _{\theta} v(\theta, p)$

$v(\theta, p)=E\left\{\sum_{k \in K_{S L}} \sum_{i=1}^{n+1} P\left(Z_{k}^{+} \in S_{i k}^{+}(n(p, \hat{\theta}))\right)\right\}$

It means that FFPN will be limited by the value $p^{*}$ for any unknown $\theta$.

\section{Numerical example}

Here we consider only the problem of aircraft fleet. Assume that $t_{S L}=40000, w=0.99$, processing the result of full scale fatigue test we get the estimate of fatigue crack parameters $\hat{\theta}=-8.5885$, the standard deviation of $\log (Q)$ is equal to $0.346, \alpha=0.286 \mathrm{~mm}$, and let for considered inspection technology $a_{d}=20 \mathrm{~mm}, a_{c}=237 \mathrm{~mm}$ (see fatigue crack in Fig. 2.32 in [1]).

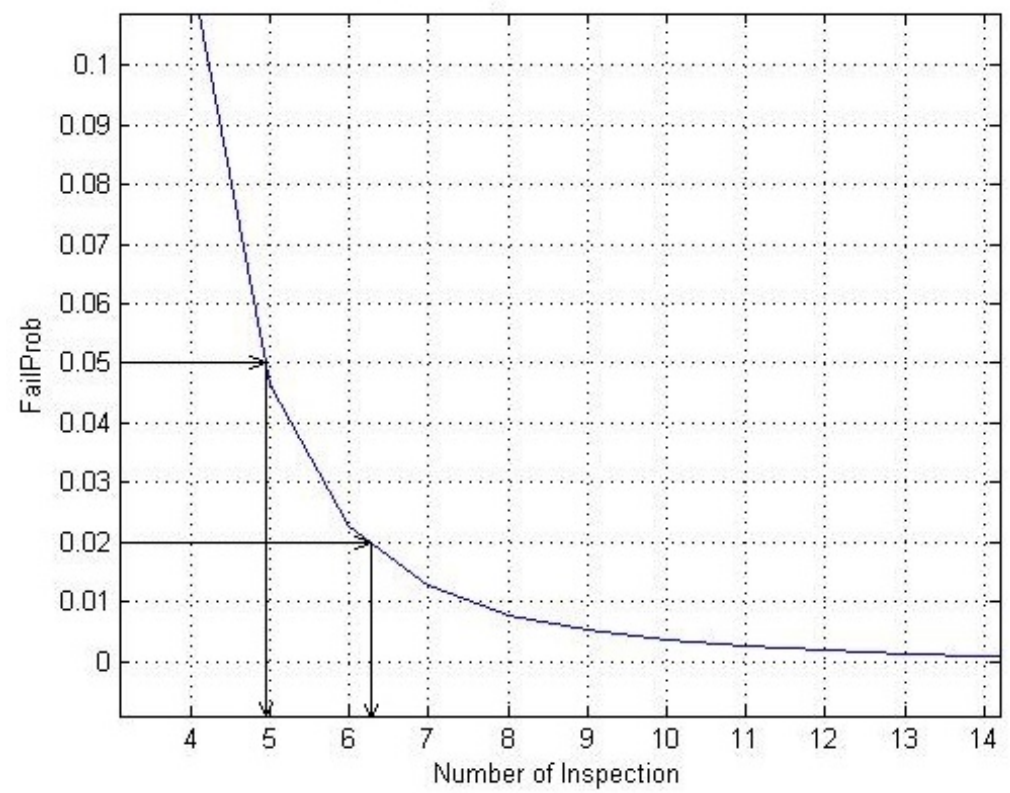

Figure 4. Example of function $p_{f N W}(n, \theta)$ (probability of crack detection ) for $\theta_{0}=-8.5885$

There are 10 aircraft in the fleet, the interval between the aircraft put in operation $d_{t}=250$; the allowed failure probability $p^{*}=0.05$, the set $\Theta_{0}$ is defined by the condition : if $\hat{n}=n(0.05, \hat{\theta})>20$ then the redesign of AC should be done. Using the Monte Carlo calculation, we get $\hat{n}=n(0.05, \hat{\theta})=5$, see Fig.4.

But this calculation is correct only if in the service the same value of $\theta_{0}$ takes place. In reality we do not know the $\theta_{0}$. For such case we should limit the maximal possible failure probability for any $\theta_{0}$. It can be done by the choice of a specific "designed" failure probability. The family of the functions 


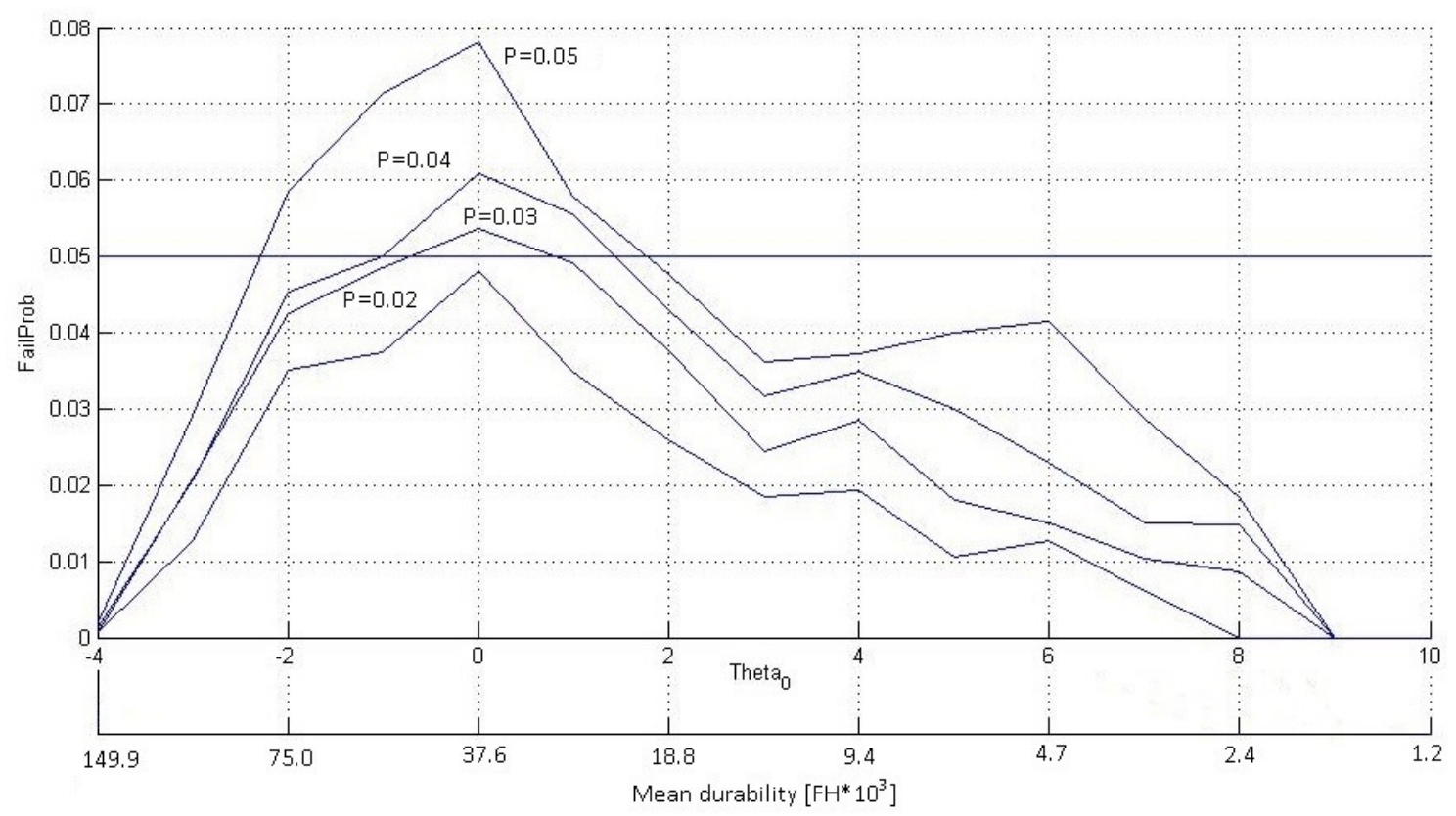

Figure 5. The function $\mathrm{v}(\theta, \mathrm{p})$ for different $\mathrm{p}$. In parallel axis the flight hours $\left(\mathrm{FH}^{*} 10^{3}\right)$ of the corresponding "Mean durability" $=$ $C_{C} / Q$ are given for $C_{C}=\log a_{c}-\log \alpha, Q=\exp \left(\theta_{0}\right)$

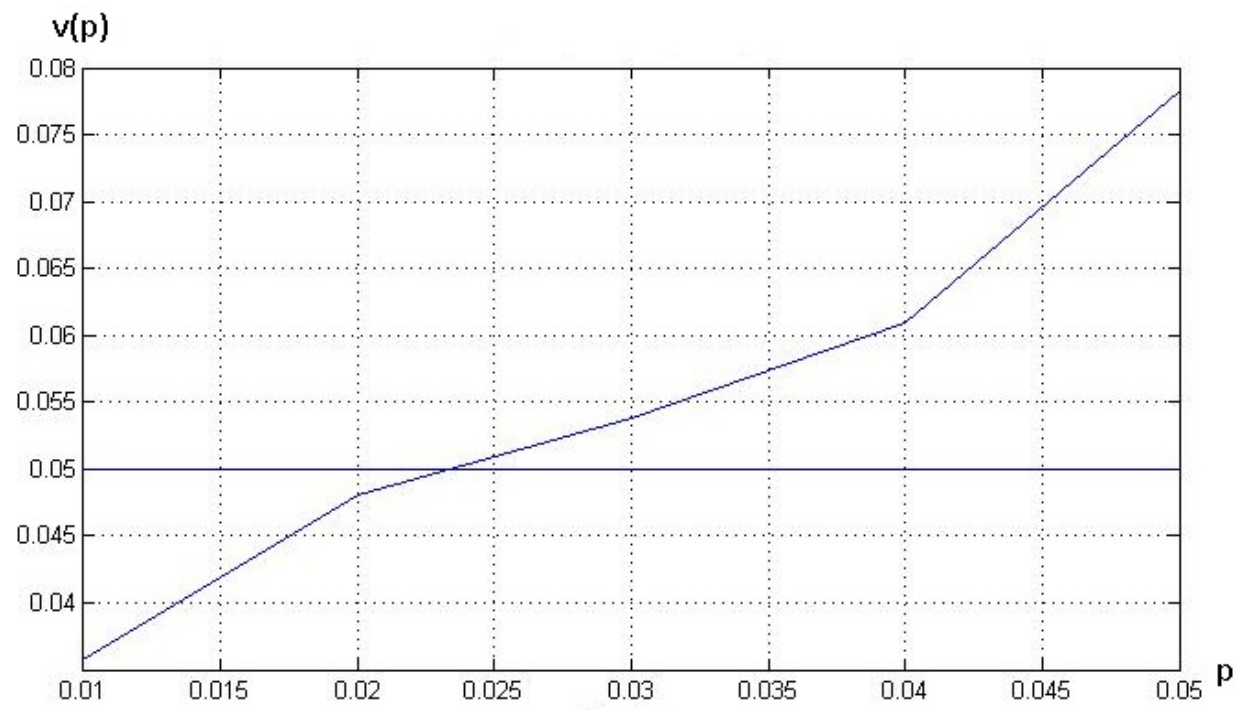

Figure 6. The function v(p)

$v(\theta, p)$ for different $p$ is shown in Fig 5, where the corresponding calculations for parallel axis are done for the corresponding "Mean durability" " $=C_{C} / Q$ for $C_{C}=\log a_{c}-\log \alpha, Q=\exp \left(\theta_{0}\right)$,

In Fig.6 the function v(p) is shown for the considered example data. We see that in order to limit FFPN by the value $p^{*}=0.05$ the value $p_{f D}=0.02$ should be chosen. Now, using the function $p_{f N W}(n, \theta)$ which is shown in Fig. 4 for the test estimate of the fatigue crack parameters $\hat{\theta}=-8.5885$, the number of inspections should be chosen to be equal to $\hat{n}=n(0.02, \hat{\theta})=7$. More complex examples of the inspection planning can be found in [1-3]. 


\section{Conclusions}

It has been found, how, with the use of the estimate of the unknown parameter $\hat{\theta}$ (after the acceptance fatigue test), one of the two decisions should be chosen: 1) to do the redesign of a new type of $\mathrm{AC}$ if the result of the test is "too bad" or 2) to make a choice of the number of inspections $n=n\left(p_{f D}, \hat{\theta}\right)$ as the function of $\hat{\theta}$ and a specific $p_{f D}$. In this case the required reliability can be provided for any unknown parameter $\theta$ and under this condition the maximum of the economic efficiency can be reached.

\section{References}

1. Paramonov, Yu., Kuznetsov, A., Kleinhofs, M. (2011) Reliabilty of fatigue-prone airframes and composite materials. Riga. RTU. 2011. 122 p.

2. Hauka, M., Paramonov, Yu. (2011) Inspection program development using minimax method. In: Proceedings of the Conference 'Relstat, 2011', Reliability and Statistics in Transportation and Communication 19-22 October 2011, pp. 105-114.

3. Paramonov, Yu., Tretyakov, S. (2012) Reliability of fleet of aircraft taking into account the information exchange about the fatigue crack discovery and human factor. Aviation. Vol. 16, No 4, 2012, pp. 103-108. 